\title{
A Long Temporal Study of Parasitism in Asexual-Sexual Populations of Carassius gibelio: Does the Parasite Infection Support Coevolutionary Red Queen Dynamics?
}

\author{
Tomáš Pakosta, ${ }^{1}$ Lukáš Vetešník, ${ }^{2}$ and Andrea Šimková $\mathbb{D}^{1}$ \\ ${ }^{1}$ Department of Botany and Zoology, Faculty of Science, Masaryk University, Kotlářská 2, 61137 Brno, Czech Republic \\ ${ }^{2}$ Institute of Vertebrate Biology, Academy of Sciences of the Czech Republic, v.v.i., Kvètná 8, 60365 Brno, Czech Republic \\ Correspondence should be addressed to Andrea Šimková; simkova@sci.muni.cz
}

Received 15 December 2017; Accepted 11 February 2018; Published 11 March 2018

Academic Editor: Yann Quilichini

Copyright (C) 2018 Tomáš Pakosta et al. This is an open access article distributed under the Creative Commons Attribution License, which permits unrestricted use, distribution, and reproduction in any medium, provided the original work is properly cited.

Carassius gibelio is an extraordinary cyprinid species exhibiting both sexual and asexual reproduction. We hypothesized that parasitism selection is one of the potential mechanisms contributing to the coexistence of the two reproductive forms of $C$. gibelio living in the same habitat. We performed a four-year study to investigate the dynamics of parasite infection in C. gibelio. According to the Red Queen prediction, the asexual form is a target of parasite adaptation due to its low genetic variability. Both sexual and gynogenetic forms of $C$. gibelio exhibited similar levels of prevalence, with monogeneans being the most frequently observed parasite group. We observed the temporal dynamics of parasite infection in the last year of investigation, when both forms were more strongly parasitized. The sexual form was more parasitized by ectoparasites in the first and last years and less parasitized by nematodes in the last year when compared to the gynogenetic form. We found no trend of high parasite infection in gynogenetic mtDNA haplotypes. We conclude that Red Queen dynamics is not the mechanism driving parasite infection in sexual-gynogenetic C. gibelio over a long time scale. Alternatively, we suggest that the dynamics of parasite infection in this complex may be generated by multiple mechanisms.

\section{Introduction}

The coexistence of sexual and asexual forms is rarely reported in vertebrates. The stable coexistence of these two forms may be achieved if there are some disadvantages for asexuals compensating the evolutionary twofold costs of sexual reproduction $[1,2]$. The coexistence of asexual and sexual reproduction is documented only in a small number of freshwater fishes including nine genera [3]. In the context of asexual reproduction, gynogenesis has a specific constraint as the sperm of males are necessary to induce embryogenesis; that is, the eggs of gynogenetic females are stimulated by sperm of usually conspecific (or, alternatively, phylogenetically closely related) males to start embryogenesis but the sperm do not fuse with the egg nucleus. Thus, in such case, the asexual and sexual forms are forced to coexist as the persistence of the sexual form is in the direct interest of the gynogenetic form.
This means that gynogenetic form (which may be considered as a "parasite" using the sperm of sexual males) cannot outcompete the sexual form (which may be considered as "host" for gynogenetic form).

The Carassius auratus complex is one of the rare complexes exhibiting both gynogenetic and sexual reproduction. This nonnative species complex colonized Europe in the second half of the 20th century and is considered to be the most successful invasive species in European waters [49], very effectively expanding and adapting to new habitats due to its high ecological tolerance [10]. Representatives of the C. auratus complex entered the Czech hydrological system by means of migration from the Danube in around $1975[7,11,12]$, and over the next 20 years the complex spread to the Danube tributaries, that is, the Morava and Dyje Rivers, where it produced stable populations [13]. The first populations of the $C$. auratus complex were triploid 
gynogenetic females $[14,15]$. Fifteen years after the initial invasion, however, mixed diploid-polyploid populations, including sexual diploid males and females, gynogenetic triploid females, and rarely triploid males or even tetraploid specimens, were reported [16-18]. In recent times, both reproductive forms have coexisted successfully in the same habitats. The particular success of colonization by former unisexual gibel carp populations was the result mainly of gibel carp's specific biological properties, that is, the ability to reproduce gynogenetically, resulting in high reproductive output, but was also due to its own invasive activities, favourable environmental factors, and human activity [14]. It seems that the shift from gynogenesis to sexual reproduction followed the stabilization of $C$. gibelio populations in the successfully colonized habitats. In the area of the Czech Republic, the $C$. auratus complex is represented by four mitochondrial lineages: C. gibelio, C. auratus, C. langsdorfii, and the so-called $\mathrm{M}$ line. Carassius gibelio is the most commonly occurring form of this complex in the Czech Republic [19].

Several mechanisms have been studied and suggested as contributing to the coexistence of sexual and asexual forms in fish complexes. Some of them have also been hypothesized and examined with respect to the $C$. auratus complex. The role of parasites in maintaining the coexistence of asexual and sexual reproduction is based on the Red Queen (RQ) hypothesis, which predicts antagonistic coevolutionary hostparasite interactions leading to sustained oscillations in their genotype frequencies [20, 21]. Following the RQ hypothesis, the asexual form is, due to its low genetic recombination, a target of parasite adaptation [22], as parasites adapt quickly to common genotypes [23]. In contrast, the sexual form quickly and effectively produces combinations of parasiteresistant genes and therefore is able to escape infection [22, 24]. In accordance with the RQ hypothesis, Hakoyama et al. [25] showed that parasitism plays an important role in maintaining the coexistence of sexual and gynogenetic forms of the $C$. auratus complex. They reported higher prevalence of the trematode species Metagonimus sp. in gynogenetic females when compared to diploid females in natural habitats. In accordance with this observation, Hakoyama and Iwasa [26] concluded that the coexistence of sexual and gynogenetic forms of the C. auratus complex is stabilized by parasites. However, the mechanisms predicted by the RQ hypothesis did not explain the coexistence of sexual and asexual forms in other freshwater fish complex, that is, Cobitis taenia hybrid complex [27]. In that case, differential habitat preferences proposed by niche shift hypothesis contributed to the coexistence of both forms. In addition, Hakoyama and Iwasa [26] also proposed that the coexistence of $C$. auratus complex is not strictly maintained by parasites but may result from interactions between several factors (i.e., assortative mating, female-biased sex allocation, different dispersion capacities, and different utilization of niches). Šmková et al. [28] examined the parasite load in gynogenetic and sexual C. gibelio in the light of the RQ hypothesis. They applied the genotyping of major histocompatibility complex (MHC) IIB genes and showed that the most common MHC genotype of the gynogenetic form is more parasitized than sexual genotypes or rare gynogenetic genotypes. In addition,
Šimková et al. [29] hypothesized that the different investments in condition-, growth-, and fitness-related traits may represent other mechanisms contributing to the coexistence of gynogenetic and sexual forms of the C. auratus complex. However, they revealed similar growth, expressed in terms of body size, and similar condition factors in gynogenetic and sexual forms and failed to identify the reproductive disadvantage for the gynogenetic form when investment in reproduction was measured by gonad weight and estradiol level. This indicates that similar energy is invested in the reproduction of gynogenetic and sexual females. In addition, Šimková et al. [29] proposed the existence of a new mechanism potentially contributing to the coexistence of gynogenetic and sexual forms of the C. auratus complex, that is, the low aerobic performance in gynogens, which may represent a physiological disadvantage balancing the evolutionary cost of sexual reproduction. In addition, Vetešník et al. [30] identified higher concentrations of triacylglycerols and cholesterol in the blood plasma of gynogenetic females, indicating a higher metabolic rate and higher energy intake when compared to sexuals.

Finally, the role of immunity in maintaining the coexistence of sexual and gynogenetic forms of the C. auratus complex was examined. While Hakoyama et al. [25] showed lower nonspecific immunity and higher parasite loads of nonspecific trematode parasite species (Metagonimus sp.) in the gynogenetic form of the $C$. auratus complex (their study was performed using gynogenetic C. langsdorfii and an unspecified sexual line of $C$. auratus complex), Šimková et al. $[28,29]$ showed no difference in nonspecific immunity between the gynogenetic and sexual forms of C. gibelio, but higher specific immunity (IgM level) as well as higher parasite loads of host specific monogeneans (Dactylogyrus spp.) in the gynogenetic form when compared to the sexual form.

As the pattern of parasite infection in fish populations composed of asexual and sexual forms had not yet been studied over a long time scale, we focused this study on the temporal dynamics of parasite infection. The aim was to perform a long-term study (over four consecutive years) to investigate the composition of parasite communities and parasite load in asexual and sexual forms of C. gibelio.

\section{Materials and Methods}

2.1. Fish Sampling. A total of 203 individuals of the Carassius auratus complex (standard length $28.35 \pm 2.61 \mathrm{~cm}$ ) were caught by electrofishing in the River Dyje near the city of Břeclav ( $48^{\circ} 80^{\prime} \mathrm{N} ; 16^{\circ} 84^{\prime} \mathrm{E}$; the Morava River basin). Fish were sampled during the same period in each of four consecutive years (first two weeks in August, 2012-2015). After blood and fin sampling, fish were immediately placed in a tank containing original water and transported to the laboratory. For each specimen, the total and standard lengths, body weight, and sex were recorded. The complete parasitological dissection of fish was performed following Ergens and Lom [31]. A fin clip of each specimen was preserved in $90 \%$ ethanol for molecular analyses. 
2.2. Parasite Collection and Identification. Monogeneans were removed from the gills or fins, placed into a drop of water on a slide, covered with a coverslip, and fixed using glycerine ammonium picrate (GAP) for later analysis [32]. The remaining parasite species were fixed in $70 \%$ ethanol. Parasite specimens were examined using an Olympus model BX50 light microscope (including an Olympus DP71 digital microscope camera) with phase contrast, differential interference contrast, and digital imaging (MicroImage 4.0 for Windows).

\subsection{Ploidy Determination and Molecular Analyses. A fin clip} of about $1 \mathrm{~cm}^{2}$ in area was taken from each fish for ploidy detection and fixed in $70 \%$ ethanol. Before analysis, this tissue was homogenised on a Petri dish in a $2 \mathrm{ml}$ solution of CyStain DNA 1 step Partec, and relative DNA content was estimated using a Partec CCA I continuous flow cytometer (Partec $\mathrm{GmbH}$; https://www.sysmex-partec.com) as was applied previously for ploidy measurements using blood sample $[33,34]$. Fresh blood of diploid C. auratus was obtained by puncture of the caudal blood vessel using a heparinised syringe and was used as a reference standard. Overall, for our study, 112 diploid specimens (comprising 56 females and 56 males) and 91 triploid females were randomly selected. The control region of mitochondrial DNA (D-loop) was analysed for each specimen. DNA was extracted by Dneasy ${ }^{\mathrm{TM}}$ Blood and Tissue Kit (Qiagen) from fin clips preserved in ethanol. PCR and DNA sequencing were performed according to Sambrook et al. [35] with small modifications following Vetešník et al. [36] and Papoušek et al. [37]. For amplification, the forward primer CarU32 ( $5^{\prime}$-CCAAAGCCAGAATTCTAAAC- $\left.3^{\prime}\right)$ and the reverse primer CarL509 ( $5^{\prime}$-CATGTGGGGTAATGA- ${ }^{\prime}$ ) [37] were used. A PCR product of 475-493 bp in length covers all ETAS domains (representing a high degree of variability considered useful for the study of intra- and interspecies evolution) [38] and a part of the central domain in the control region. PCR was performed in a final volume of $30 \mu \mathrm{l}$ containing 1x PCR reaction buffer, $1.5 \mathrm{mM} \mathrm{MgCl}_{2}, 0.3 \mu \mathrm{M}$ of each primer (CarU32 and CarL509), $0.2 \mathrm{mM}$ dNTPs, $1.5 \mathrm{U}$ Taq polymerase, and $100 \mathrm{ng} / \mu \mathrm{l}$ of template gDNA. PCR was carried out using the following steps: $1 \mathrm{~min}$ of denaturation at $95^{\circ} \mathrm{C}$, followed by 32 cycles of $45 \mathrm{~s}$ at $94^{\circ} \mathrm{C}, 30 \mathrm{~s}$ at $50^{\circ} \mathrm{C}$, and $45 \mathrm{~s}$ at $72^{\circ} \mathrm{C}$, and $10 \mathrm{~min}$ of final elongation at $72^{\circ} \mathrm{C}$. The PCR products were checked on $1.5 \%$ agarose gel, purified by High Pure PCR product purification kit (Roche), and directly sequenced using BigDye Terminator Cycle sequencing kit on an ABI 3130 Genetic Analyser (Applied Biosystems, USA). The sequences were aligned using MEGA 6.0 [39] and analysed using TCS 1.21 [40]. The obtained sequences were compared with the sequences for the mtDNA haplotypes of the C. auratus complex available in GenBank. The sequences of mtDNA haplotypes obtained in this study were identical with the sequences available in GenBank under the following accession numbers: FJ167410, FJ167411, FJ167413, FJ167414, FJ167416, FJ167420, and FJ167423-FJ167425.

2.4. Statistical Analyses. General linear models (GLM) were used to evaluate the three main effects, that is, ploidy, sex, and year, on parasite abundance, as well as two interaction effects, that is, ploidy with year and ploidy with body size. The standard body length of fish was included as a covariate. Parasite abundance was expressed by the following variables: total parasite abundance (i.e., the abundance including all parasite specimens), total monogenean abundance (i.e., the abundance of the most common parasitic group), total abundance of Nematoda (i.e., the abundance of the most common endoparasite group), and the abundance of the most common parasitic species or genera, that is, Dactylogyrus spp. (gill Monogenea), Gyrodactylus spp. (gill and fin Monogenea), and Ichthyophthirius multifiliis (Protozoa). For multiple comparisons, the Tukey post hoc test was used. All variables applied in this study were examined for normal distribution and homogeneity of variance. For data that did not fit these assumptions log-transformation was applied. Statistical analyses were performed in Statistica for Windows 12, StatSoft Inc.

\section{Results}

Random sampling of the mixed population of C. gibelio in the studied locality over a period of four years revealed the following composition of the mixed gynogenetic-sexual population: $38 \%$ of fish were triploid specimens ( $37 \%$ were gynogenetic females and $1 \%$ were triploid males) and $63 \%$ of fish were sexual diploid specimens. Molecular analysis of mtDNA confirmed that all specimens were representatives of the $C$. auratus complex. Our study revealed the presence of 9 variants of $16 \mathrm{mtDNA}$ haplotypes previously described in the Southern Moravian region of the Czech Republic by Papoušek [19] (Table 1). The Carassius gibelio lineage was the most frequent in the studied locality (96\%). Six mtDNA haplotype variants of C. gibelio were identified: G01, G02, G04, G05, G07, and G11, with G02 representing the most common haplotype (Table 1). Two of the other haplotypes, G01 and G04, were also common. For sexual specimens, G02 and G04 were the most common haplotypes and G05 was a rare mitochondrial genotype in all four years of investigation. For gynogenetic females, differences in haplotype frequencies among the four years were observed. The most frequent haplotypes of gynogenetic females reported in each year were as follows: G01, G02, and G07 in 2012; G02 in 2013; and G01 in both 2014 and 2015. Among the 6 C. gibelio mtDNA haplotypes, 3 haplotypes were found exclusively in gynogenetic females, one was found exclusively in sexual specimens, and two were shared by sexual and gynogenetic specimens. The other three lineages, C. auratus (haplotype A01), C. langsdorfii (haplotype L01), and M line (haplotype $\mathrm{M} 01)$, were rare $(2.4 \%, 0.5 \%$, and $1 \%$, resp.). For the following analyses, only the C. gibelio lineage was selected due to the high frequency of this form.

Parasite communities consisted of the representatives of 8 taxonomic groups (Table 2). A total of 11 species of Monogenea, 1 species of Cestoda, 3 species of Trematoda, 2 species of Nematoda, 1 species of Hirudinea, 1 species of Mollusca (larval stages termed glochidium), 1 species of Protozoa, and 2 species of Crustacea were identified (Table 2). The parasite infection level (expressed by prevalence, abundance, and intensity of infection) was compared between 
TABLE 1: Haplotypes of the mitochondrial control region (D-loop) in investigated specimens of Carassius auratus complex. Total numbers of specimens and the numbers of specimens in each of four consecutive years (in parentheses) are shown for each mtDNA haplotype.

\begin{tabular}{|c|c|c|c|}
\hline C. auratus complex & Haplotype & Number of diploids & Number of triploids \\
\hline \multirow{6}{*}{ C. gibelio } & G01 & - & $43(11 / 4 / 14 / 14)$ \\
\hline & G02 & $61(16 / 21 / 15 / 9)$ & $27(7 / 8 / 5 / 7)$ \\
\hline & G04 & $34(10 / 9 / 6 / 9)$ & $2(0 / 1 / 0 / 1)$ \\
\hline & G05 & $9(1 / 2 / 5 / 1)$ & - \\
\hline & G07 & - & $11(8 / 2 / 0 / 1)$ \\
\hline & G11 & - & $8(4 / 3 / 1 / 0)$ \\
\hline C. auratus & A01 & $5(3 / 1 / 1 / 0)$ & - \\
\hline C. langsdorfii & L01 & $1(0 / 0 / 1 / 0)$ & - \\
\hline C. auratus M line & M01 & $2(0 / 1 / 0 / 1)$ & - \\
\hline
\end{tabular}

gynogenetic and sexual forms. The prevalence of Dactylogyrus spp. and Gyrodactylus sprostonae (Monogenea) was high in sexual males and females as well as in gynogenetic females (maximum prevalence was reported for 5 Dactylogyrus species and a prevalence of more than $80 \%$ for Gyrodactylus). More specifically, Dactylogyrus dulkeiti, D. intermedius, D. anchoratus, D. formosus, and D. inexpectatus and Gyrodactylus sprostonae represented the ectoparasite species reaching the highest intensities of infection in both forms. The prevalence of 5 other ectoparasite species, that is, D. vastator, D. baueri, G. shulmani, G. longoacuminatus, and Ichthyophthirius multifiliis, was moderate. However, the intensity of infection of I. multifiliis was also high. The prevalence of other parasite species was low $(\leq 45 \%)$. Concerning endoparasite species, Schulmanela petruschewskii and Philometroides sanguinea (Nematoda) achieved the highest intensity of infection, especially in females (both gynogenetic and sexual). Total parasite abundance as well as monogenean parasite abundance was lower in gynogenetic G07 haplotypes when compared to other haplotypes (Mann-Whitney test, $p=0.018$ and $p=0.008$, resp.).

The effects of ploidy, sex, and year on parasite abundance were analysed (Table 3) as described in Materials and Methods. Total parasite abundance (Figure 1(a)) as well as monogenean abundance (Figure 1(b)) was significantly affected by year, body size, and ploidy (through the interaction effect of ploidy and year). The highest levels of total parasite abundance and monogenean abundance were reported in 2015 , the lowest in 2013. Sexual diploids were more parasitized than gynogenetic females in $2012(p<0.05)$ and $2015(p<$ $0.01)$. However, no significant differences in total parasite abundance or monogenean abundance were found between 2013 and 2014.

Dactylogyrus abundance was significantly affected by year and body size and there was also a weak but insignificant effect of ploidy ( $p=0.127)$ through the interaction effect of ploidy and year (Figure 1(c), Table 3). Dactylogyrus abundance was significantly higher in 2015 compared to the first two years of investigation and was also higher in 2014 compared to 2013. In 2015, Dactylogyrus abundance was slightly higher in sexual diploids than in gynogenetic females $(p=0.046)$. Gyrodactylus abundance (Figure $1(\mathrm{~d}))$ was significantly affected by year and ploidy through the interaction effect of ploidy and year. Gyrodactylus abundance reached its highest values in 2015 and was also higher in 2012 compared to 2013. In 2012 and 2015, sexual diploids were more parasitized by Gyrodactylus spp. when compared to gynogenetic females $(p<0.01)$.

The abundance of $I$. multifiliis was significantly affected by year and body size, and there was a small but insignificant effect of ploidy ( $p=0.14$ ) through the interaction effect of ploidy and year (Table 3, Figure 1(e)). The lowest abundance of I. multifiliis was found in 2012. In 2012 and 2015, sexual diploids were more parasitized by $I$. multifiliis when compared to gynogenetic females.

The abundance of Nematoda (two nematode species were pooled for the analysis) was significantly affected by year, ploidy, and body size (Table 3, Figure 1(f)). A significant interaction effect of ploidy and body size on Nematoda abundance was also found. The highest Nematoda abundance was found in 2015 and the lowest in 2013. Overall, females were more parasitized than males $(p=0.002)$ and gynogenetic females were more parasitized than sexual diploids $(p=0.010$ as evidenced in 2014 and 2015; see Figure 1(f)). However, when the effect of ploidy was analysed within each year, a significant difference in the abundance of Nematoda between gynogenetic females and sexual diploids was found only in $2015(p=0.004)$.

\section{Discussion}

The Red Queen hypothesis describes host-parasite dynamics on the basis of negative parasite mediated frequency dependent selection, which can represent one of the advantages of sexual reproduction over asexual reproduction $[2,22$, 41, 42]. Thus, we may expect that the asexual form of a species, which exhibits low genetic variability, will suffer from higher parasite load when compared to the sexual form, which exhibits high genetic variability due to recombination. However, as already mentioned, there is no clear empirical support for this hypothesis, as studies focussing on parasite load in coexisting asexual and sexual complexes do not show a consistent pattern. In the case of the gynogenetic-sexual 


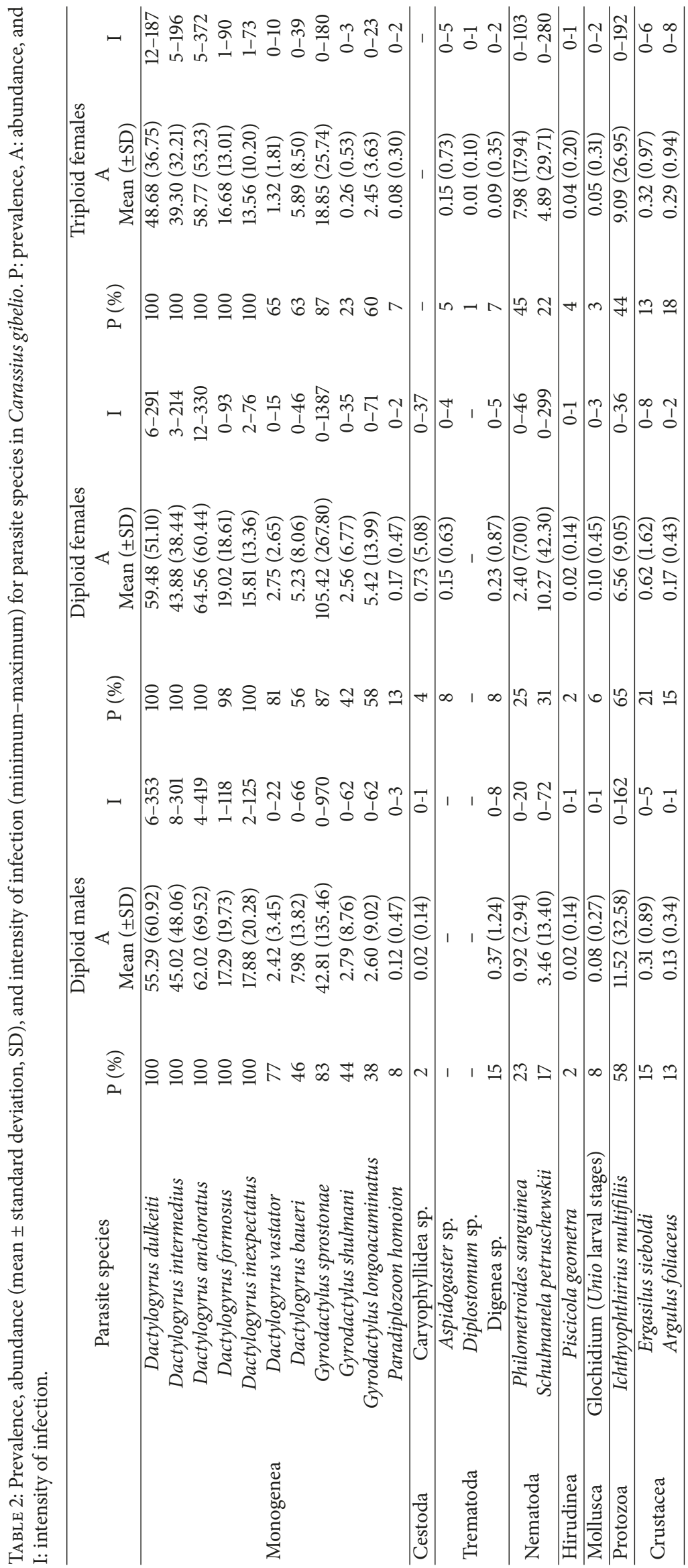


TABLE 3: The effects of ploidy, sex, year, and body size on parasite abundance (transformed in log). Parasite abundance was expressed by total parasite abundance, abundance of the most common parasitic groups, genera, or species in Carassius gibelio. The statistically significant $p$ values are shown in bold.

\begin{tabular}{|c|c|c|c|c|c|}
\hline Dependent variable & Predicted variables & $F$ & $p$ & Total $F$ & Total $p$ \\
\hline \multirow{6}{*}{ Total parasites } & Year & 14.432 & $<0.001$ & \multirow{6}{*}{10.038} & \multirow{6}{*}{$<0.001$} \\
\hline & Sex & 0.002 & 0.960 & & \\
\hline & Ploidy & 0.002 & 0.964 & & \\
\hline & Body size & 11.572 & 0.001 & & \\
\hline & Year $*$ ploidy & 3.556 & 0.015 & & \\
\hline & Ploidy $*$ body size & 0.001 & 0.979 & & \\
\hline \multirow{6}{*}{ Monogenea } & Year & 13.720 & $<0.001$ & \multirow{6}{*}{9.119} & \multirow{6}{*}{$<0.001$} \\
\hline & Sex & 0.010 & 0.922 & & \\
\hline & Ploidy & 0.206 & 0.651 & & \\
\hline & Body size & 6.660 & 0.011 & & \\
\hline & Year $*$ ploidy & 4.063 & 0.008 & & \\
\hline & Ploidy $*$ body size & 0.279 & 0.598 & & \\
\hline \multirow{6}{*}{ Dactylogyrus } & Year & 8.387 & $<0.001$ & \multirow{6}{*}{5.530} & \multirow{6}{*}{$<0.001$} \\
\hline & Sex & 0.482 & 0.488 & & \\
\hline & Ploidy & 0.051 & 0.822 & & \\
\hline & Body size & 6.851 & 0.010 & & \\
\hline & Year $*$ ploidy & 1.927 & 0.127 & & \\
\hline & Ploidy $*$ body size & 0.069 & 0.794 & & \\
\hline \multirow{6}{*}{ Gyrodactylus } & Year & 18.599 & $<0.001$ & \multirow{6}{*}{9.557} & \multirow{6}{*}{$<0.001$} \\
\hline & Sex & 0.110 & 0.740 & & \\
\hline & Ploidy & 0.038 & 0.846 & & \\
\hline & Body size & 1.446 & 0.231 & & \\
\hline & Year $*$ ploidy & 4.489 & 0.005 & & \\
\hline & Ploidy $*$ body size & 0.073 & 0.787 & & \\
\hline \multirow{6}{*}{ Ichthyophthirius multifiliis } & Year & 11.887 & $<0.001$ & \multirow{6}{*}{8.268} & \multirow{6}{*}{$<0.001$} \\
\hline & Sex & 0.210 & 0.647 & & \\
\hline & Ploidy & 0.151 & 0.698 & & \\
\hline & Body size & 8.936 & 0.003 & & \\
\hline & Year $*$ ploidy & 1.847 & 0.140 & & \\
\hline & Ploidy $*$ body size & 0.191 & 0.662 & & \\
\hline \multirow{6}{*}{ Nematoda } & Year & 12.201 & $<0.001$ & \multirow{6}{*}{8.217} & \multirow{6}{*}{$<0.001$} \\
\hline & Sex & 1.884 & 0.172 & & \\
\hline & Ploidy & 7.624 & 0.006 & & \\
\hline & Body size & 14.008 & $<0.001$ & & \\
\hline & Year $*$ ploidy & 1.191 & 0.315 & & \\
\hline & Ploidy $*$ body size & 7.690 & 0.006 & & \\
\hline
\end{tabular}

complex including sexual Poeciliopsis monacha and the two coexisting gynogenetic triploid clones of $P$. 2monacha-lucida, Lively et al. [22] reported a higher accumulation of the metacercaria stage of the trematode Uvulifer sp. causing black spot disease in the most common triploid clone when compared to sexual species and the less common gynogenetic clone on different temporal and spatial scales (i.e., two different years of collection and three different pools). Infection by the metacercaria stage of the trematode Metagonimus sp., also forming a black spot on the fish epidermis, was analysed in the gynogenetic-sexual complex of Carassius auratus langsdorfii-C. a. bürgeri by Hakoyama et al. [25]. In this complex, the gynogenetic form is infrequent. They suggested that the higher parasite load in the gynogenetic form was, in part, due to the lower nonspecific immune reaction in the gynogenetic form compared to the sexual form. In contrast, no consistent pattern in parasite load between asexual Poecilia formosa and sexual P. latipinna was observed on the spatial scale [43]. In their study, four populations were studied-two exhibiting no difference in parasite load between asexual and sexual species, one population exhibiting high overall parasite load among asexual species, and the last exhibiting a greater micro-parasite load among sexual species.

In our study, we revealed the same composition of parasite communities in both sexual and gynogenetic forms of $C$. gibelio, that is, a high number of parasite species with Monogenea (ectoparasite group exhibiting generally high degree of host specificity) predominant and similar levels of prevalence. 


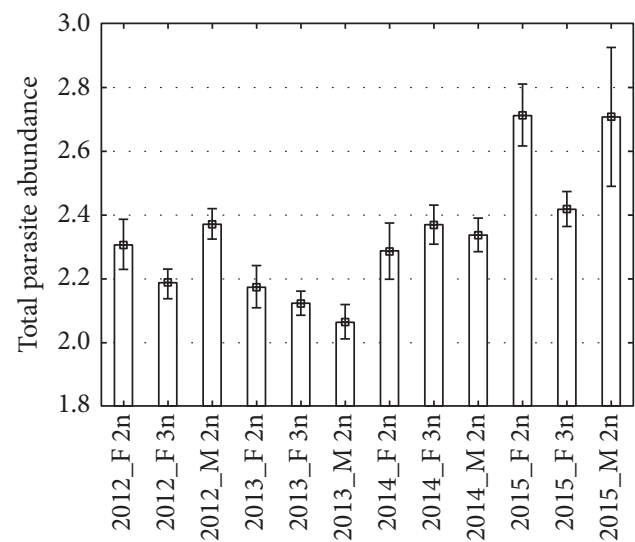

(a) Mean

I Mean $\pm \mathrm{SE}$

(a)

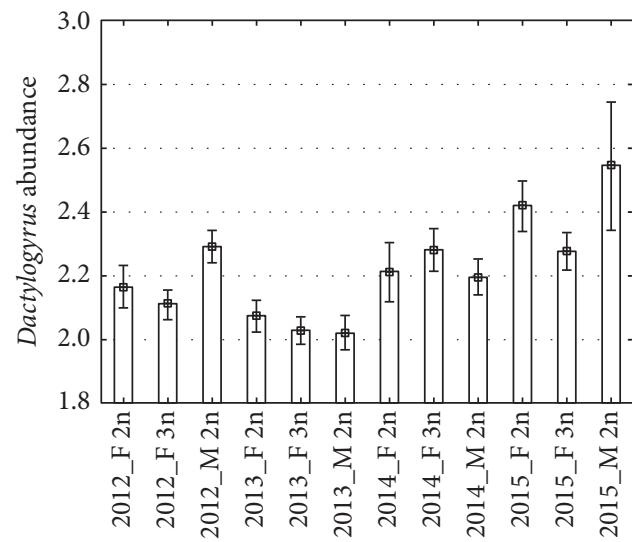

回 Mean

I Mean $\pm \mathrm{SE}$

(c)

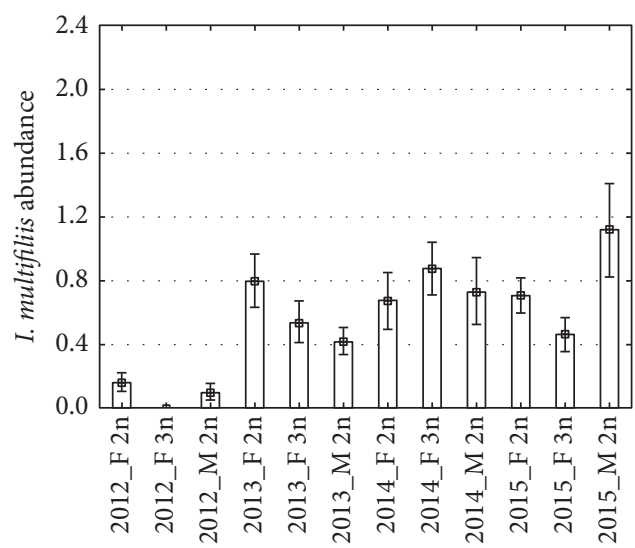

回 Mean

I Mean $\pm \mathrm{SE}$

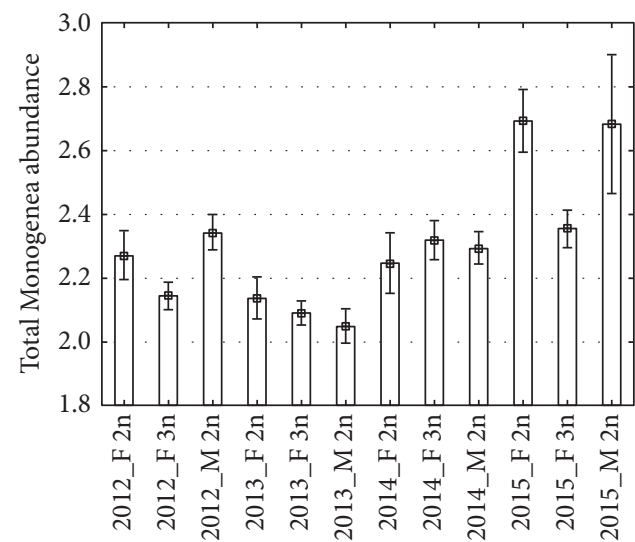

回 Mean

I Mean \pm SE

(b)

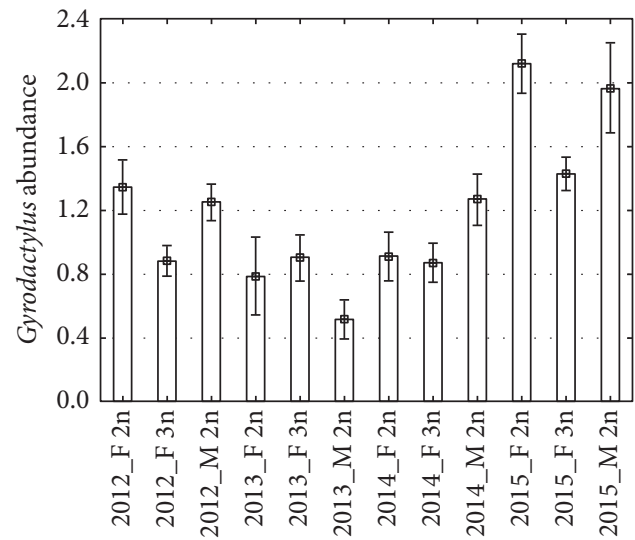

回 Mean

I Mean $\pm \mathrm{SE}$

(d)

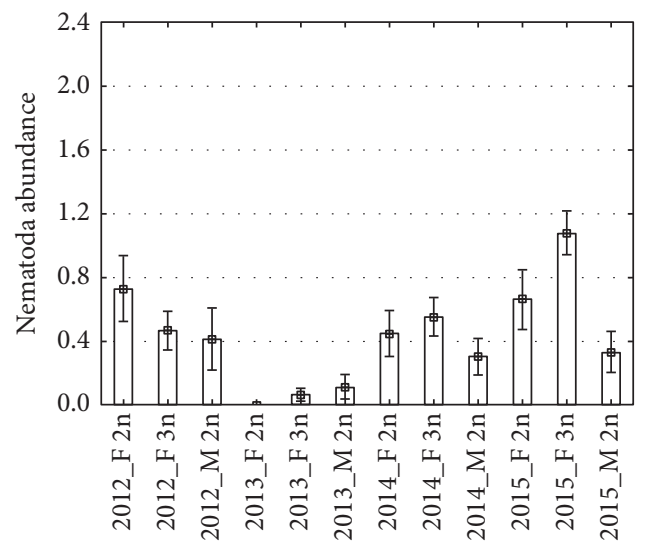

回 Mean

I Mean $\pm \mathrm{SE}$

(e)

(f)

FIGURE 1: Parasite abundance (transformed in log) in sexual diploid form (F2n: diploid females and M2n: diploid males) and gynogenetic triploid females (F3n). Parasite abundance is expressed using the total parasites (a), Monogenea (b), Dactylogyrus species (c), Gyrodactylus species (d), Ichthyophthirius multifiliis (e), and Nematoda (f). 
Surprisingly, some monogenean species exhibited a trend of higher maximum intensity of infection in sexual specimens when compared to gynogenetic females. This trend was most evident for Gyrodactylus sprostonae, representing monogeneans with a viviparous strategy (and very fast reproduction rate) and lower degree of host specificity when compared to oviparous and highly host specific gill monogeneans of Dactylogyrus. On the other hand, gynogenetic females tended to be more parasitized with respect to the total number of nematodes (but see below). However, within-year comparisons revealed higher monogenean abundance (for both Dactylogyrus and Gyrodactylus species) in the sexual form when compared to the gynogenetic form partially for 2012 and particularly for 2015, when the overall level of parasite infection was the highest. No obvious differences in monogenean abundance were found in 2013, and, in 2014, Dactylogyrus abundance among gynogenetic females even tended to be slightly higher than Dactylogyrus abundance among the sexual form (though the difference was not significant). Concerning endoparasites, Nematoda were the most abundant parasite group, with two species reported in this study-Philometroides sanguinea (parasite species restricted to Carassius species) and Schulmanela petruschewskii (parasitizing a wide range of fish species). The gynogenetic form was more parasitized by nematodes than the sexual form. However, it seems that this result was generated by the effect of body size, as gynogenetic females reached greater body sizes than sexual specimens. During all four years of investigation, gynogenetic females represented $30-40 \%$ of the mixed population; sexual females, $35-40 \%$; and sexual males, $22-30 \%$. As a result, the sexual form was more frequent in the habitat than the asexual form, which may partially explain the observed pattern of parasite infection. The coexisting gynogenetic-sexual C. gibelio complex was previously investigated by Šimková et al. [28]. They found that there was a small difference in parasite load between sexual and gynogenetic forms (gynogenetic females suffered from slightly higher parasite infection). However, in their study, the selected mixed diploid-triploid population of C. gibelio was collected at two-time points (two consecutive years), with coexisting gynogenetic females representing $51-54 \%$ of the population, sexual females representing $20-24 \%$, and sexual males representing $20-22 \%$. A different ratio of sexual and asexual forms of $C$. gibelio in a given population, a different population density, or the influence of unexamined abiotic or biotic factors may potentially explain the difference between the results observed in the present study and those obtained previously by Šimková et al. [28], as in the latter study the sampling of C. gibelio was performed in a different locality. In addition, in the study by Šimková et al. [28], Gyrodactylus parasites represented only a small fraction of the observed parasite communities, while Dactylogyrus parasites (with an oviparous strategy, a slower reproduction rate, and higher degree of host specificity when compared to Gyrodactylus) predominated. It is also possible that, in some localities, the coexistence of gynogenetic and sexual forms of $C$. gibelio is not solely maintained by parasites and that some other mechanisms may be involved or even act more strongly than parasites. It is also possible that phylogenetically closely related cyprinid species (i.e., Carassius carassius and Cyprinus carpio) living in sympatry with $C$. gibelio act as suitable host species for the transmission of host specific parasites in some localities. However, their occurrence was extremely rare in our system studied. The general validity of the RQ hypothesis with respect to how the coexistence of gynogenetic and sexual forms of C. gibelio is maintained needs to be verified by examining a wider range of mixed populations with different ratios of gynogenetic and sexual forms likely reflecting the specific stages of evolution of this complex.

Šimková et al. [28] also performed genotyping using the major histocompatibility complex genes (MHC IIB) of gynogenetic and sexual specimens. They hypothesized and confirmed that the most common gynogenetic clone is the target of parasite selection; that is, they showed that the most frequent MHC clones of gynogenetic C. gibelio are more parasitized than sexual specimens or gynogenetic specimens with rare $\mathrm{MHC}$ genotypes. In the present study, no data concerning MHC genotype diversity was available. Here, we applied an analysis of mitochondrial haplotype diversity (based on Dloop sequence analysis). Concerning the mtDNA haplotypes in C. gibelio in the Czech Republic previously studied by Papoušek [19], G02 was the most common. In the locality sampled for this study, G02 was also the most common haplotype reported in the total sample of C. gibelio and was more frequently reported in sexual diploids than in gynogenetic triploid females. In accordance with Papoušek [19], we identified G05 haplotype only in sexual diploids and G11 only in gynogenetic females. However, our data revealed G01 as the most frequent haplotype in gynogenetic females (47\% of specimens). In contrast to Papoušek [19], we failed to identify this haplotype in sexual diploid form. Concerning overall haplotype diversity, the gynogenetic form exhibited a higher number of haplotypes when compared to the sexual form. When comparing haplotype frequencies among the four years, similar frequencies of haplotypes were identified for the sexual form, while some changes in haplotype frequency were observed for gynogenetic females. However, these changes were not commensurate with changes in parasite infection among years. Parasite abundance was similar in all gynogenetic, sexual, and mixed haplotypes, except for one rare gynogenetic haplotype, namely, G07, which was less parasitized than other haplotypes. This less parasitized haplotype was present mainly in fish sampled in 2012.

\section{Conclusions}

In the present long-term study, we investigated the compositions of parasite communities and levels of parasite infection in coexisting gynogenetic and sexual forms of the cyprinid C. gibelio, representing the most common form of the $C$. auratus complex in the Czech Republic. Gynogenetic and sexual forms shared almost all parasite species and, overall, exhibited similar levels of parasite infection. However, the level of parasite infection was variable for both forms over the four years of investigation, potentially as a result of some temporal variability of multiple abiotic and/or biotic factors. 
We found higher mtDNA haplotype diversity in the gynogenetic form when compared to the sexual form. Our results indicate that changes in the frequencies of mitochondrial genotypes across years do not explain the observed temporal variability in parasite infection. Different patterns of parasite infection in sexual and gynogenetic forms of C. gibelio were reported when comparisons were made within individual years, a finding which may potentially be associated with the dynamics of host-parasite interactions. However, the observed dynamics of parasite infection does not support the RQ hypothesis, as we failed to find a significantly higher level of parasite infection in the asexual form in any single year of investigation. With respect to future studies focused on the dynamics of host-parasite interactions, we suggest the need to analyse other genetic markers, especially those related to host immunity.

\section{Conflicts of Interest}

The authors declare no conflicts of interest regarding the publication of this paper.

\section{Authors' Contributions}

Andrea Šimková designed the study. Tomáš Pakosta analysed the data. Lukáš Vetešník collected fish material. All authors were involved in drafting and revising the manuscript. All authors read and approved the final version of the manuscript.

\section{Acknowledgments}

The study was funded by the Czech Science Foundation, Project no. P505/12/0375. Andrea Šimková and Tomáš Pakosta were partially funded by the Czech Science Foundation, ECIP project, no. P505/12/G112. The authors are grateful to M. Benovics, L. Gettová, M. L. Červenka Kičinja, K. Koukalová, V. Krasnovyd, C. Rahmouni, and G. Vágnerová for their help with fish dissection and parasite collection and K. Civáňová and S. Stierandová for their help with molecular analyses. They would like to thank K. Halackka from the Institute of Vertebrate Biology, Academy of Sciences of the Czech Republic, for his help with fish sampling. They also thank Matthew Nicholls for English language revision of the manuscript.

\section{References}

[1] J. M. Smith, The Evolution of Sex, Cambridge University Press, Cambridge, UK, 1978.

[2] G. Bell, The Masterpiece of Nature: the Evolution and Genetics of Sexuality, University of California Press, Berkeley, Calif, USA, 1982.

[3] D. K. Lamatsch and M. Stöck, "Sperm-dependent parthenogenesis and hybridogenesis in teleost fishes," in Lost Sex, I. Schön, K. Martens, and P. Dijk, Eds., pp. 399-432, Springer, Dordrecht, Netherlands, 2009.
[4] F. W. Allendorf, "Ecological and Genetic Effects of Fish Introductions: Synthesis and Recommendations," Canadian Journal of Fisheries and Aquatic Sciences, vol. 48, no. S1, pp. 178-181, 1991.

[5] P. B. Moyle and T. Light, "Fish invasions in California: Do abiotic factors determine success?” Ecology, vol. 77, no. 6, pp. 1666-1670, 1996.

[6] I. G. Cowx, "Introduction of fish species into european fresh waters: Economic successes or ecological disasters ?" BFPP Bulletin Francais de la Peche et de la Protection des Milieux Aquatiques, no. 344-345, pp. 57-77, 1997.

[7] S. Lusk, V. Lusková, and K. Halačka, "Prussian carp - 25 years since its natural introduction," in Proceedings of the 3rd Czech Conference of Ichthyology, pp. 135-140, 1998.

[8] J. A. Szczerbowski, P. M. Banarescu, and H. J. Poepke, "The Freshwater Fishes of Europe, Cyprinidae 2, Part III, Carassius to Cyprinus," AULA-Verlag, vol. 5, no. 3, pp. 5-41, 2002.

[9] S. Lusk and V. Lusková, "Invasive fish species in the Czech Republic," in Proceedings of the 8th Czech Conference of Ichthyology, pp. 135-140, Czech, 2005.

[10] E. García-Berthou, C. Alcaraz, Q. Pou-Rovira, L. Zamora, G. Coenders, and C. Feo, "Introduction pathways and establishment rates of invasive aquatic species in Europe," Canadian Journal of Fisheries and Aquatic Sciences, vol. 62, no. 2, pp. 453463, 2005.

[11] S. Lusk, V. Baruš, and V. Veselý, "On the occurrence of Carassius auratus in the Morava river drainage area," Folia Zoologica, vol. 4, no. 26, pp. 377-381, 1977.

[12] S. Lusk, V. Luskova, and L. Hanel, "Alien fish species in the Czech Republic and their impact on the native fish fauna," Folia Zoologica, vol. 59, no. 1, pp. 57-72, 2010.

[13] S. Lusk, J. Koščo, V. Lusková, K. Halačka, and P. Košuth, "Alien fish species in the floodplains of the Dyje and the Bodrog rivers," Ecohydrology \& Hydrobiology, vol. 4, no. 2, pp. 199-205, 2004.

[14] S. Lusk, V. Baruš, and A. Kirka, "Current spreading and importance of the giebel (Carassius auratus gibelio Bloch) in Czechoslovakia," Czech Journal of Animal Science, vol. 25, no. 11, pp. 871-878, 1980 (Czech).

[15] V. Baruš and O. Oliva, Fauna of Czech Republic and Slovak Republic, Petromyzontes and Osteichtyes, Academia, Prague, Czech Republic, 1995.

[16] V. Lusková, K. Halačka, L. Vetešník, and S. Lusk, "Changes of ploidy and sexuality status of 'Carassius auratus' populations in the drainage area of the River Dyje (Czech Republic)," Ecohydrology \& Hydrobiology, vol. 4, no. 2, pp. 165-171, 2004.

[17] V. Lusková, S. Lusk, K. Halačka, and L. Vetešník, "Carassius auratus gibelio-The most successful invasive fish in waters of the Czech Republic," Russian Journal of Biological Invasions, vol. 1, no. 3, pp. 176-180, 2010.

[18] L. Vetešník, K. Halačka, V. Lusková, and S. Lusk, "Erythrocyte profile of diploid and triploid silver crucian carp (Carassius auratus)," Acta Veterinaria Brno, vol. 75, no. 2, pp. 203-207, 2006.

[19] I. Papoušek, Molecular-genetic analyses of Carassius species in central Europe [Ph.D. thesis], Masaryk University, Faculty of Science, Brno, Czech Republic, 2008.

[20] L. Van Valen, "A new evolutionary law," Evolutionary Theory, vol. 1, pp. 1-30, 1973.

[21] J. Jaenike, "An hypothesis to account for the maintenance of sex within populations," Evolutionary Theory, vol. 3, pp. 191-194, 1978. 
[22] C. M. Lively, C. Craddock, and R. C. Vrijenhoek, "Red Queen hypothesis supported by parasitism in sexual and clonal fish," Nature, vol. 344, no. 6269, pp. 864-866, 1990.

[23] K. P. Lampert, P. Fischer, and M. Schartl, "Major histocompatibility complex variability in the clonal Amazon molly, Poecilia formosa: Is copy number less important than genotype?" Molecular Ecology, vol. 18, no. 6, pp. 1124-1136, 2009.

[24] C. M. Lively and M. F. Dybdahl, "Parasite adaptation to locally common host genotypes," Nature, vol. 405, no. 6787, pp. 679$681,2000$.

[25] H. Hakoyama, T. Nishimura, N. Matsubara, and K. Iguchi, "Difference in parasite load and nonspecific immune reaction between sexual and gynogenetic forms of Carassius auratus," Biological Journal of the Linnean Society, vol. 72, no. 3, pp. 401407, 2001.

[26] H. Hakoyama and Y. Iwasa, "Coexistence of a sexual and an unisexual form stabilized by parasites," Journal of Theoretical Biology, vol. 226, no. 2, pp. 185-194, 2004.

[27] J. Kotusz, M. Popiolek, P. Drozd, K. De Gelas, V. Šlechtová, and K. Janko, "Role of parasite load and differential habitat preferences in maintaining the coexistence of sexual and asexual competitors in fish of the Cobitis taenia hybrid complex," Biological Journal of the Linnean Society, vol. 113, no. 1, pp. 220235, 2014

[28] A. Šimková, M. Košař, L. Vetešník, and M. Vyskočilová, "MHC genes and parasitism in Carassius gibelio, a diploid-triploid fish species with dual reproduction strategies," BMC Evolutionary Biology, vol. 13, article 122, 2013.

[29] A. Šimková, P. Hyršl, K. Halačka, and L. Vetešník, "Physiological and condition-related traits in the gynogenetic-sexual Carassius auratus complex: different investments promoting the coexistence of two reproductive forms?" BMC Evolutionary Biology, vol. 15, article 438, 2015.

[30] L. Vetešník, K. Halačka, and A. Šimková, “The effect of ploidy and temporal changes in the biochemical profile of gibel carp (Carassius gibelio): A cyprinid fish species with dual reproductive strategies," Fish Physiology and Biochemistry, vol. 39, no. 2, pp. 171-180, 2013.

[31] R. Ergens and J. Lom, Causative Agents of Fish Diseases, Academia, Prague, Czech Republic, 1970.

[32] G. Malmberg, "On the occurrence of Gyrodactylus on Swedish fishes," Skrifter utgivna av Sodra Sveriges Fiskeriforening, pp. 1976, 1957 (Swedish).

[33] M. Flajšhans, "A model approach to distinguish diploid and triploid fish by means of computer-assisted image analysis," Acta Veterinaria Brno, vol. 66, no. 2, pp. 101-110, 1997.

[34] K. Halačka and V. Lusková, "Polyploidy in Carassius auratus in the lower reaches of the river Dyje - determination using the size of erythrocyte nuclei," in Proceedings of the 4th Czech Ichthyological Conference, Book of Abstracts, pp. 106-109, Research Institute of Fish Culture and Hydrobiology, Vodňany, Czech Republic, 2000.

[35] J. Sambrook, E. F. Fritsch, and T. Maniatis, Molecular Cloning: A Laboratory Manual, University of Texas South Western Medical Center, Dallas, Tex, USA, 1989.

[36] L. Vetešník, I. Papoušek, K. Halačka, V. Lusková, and J. Mendel, "Morphometric and genetic analysis of Carassius auratus complex from an artificial wetland in Morava River floodplain, Czech Republic," Fisheries Science, vol. 73, no. 4, pp. 817-822, 2007.

[37] I. Papoušek, L. Vetešník, K. Halačka, V. Lusková, M. Humpl, and J. Mendel, "Identification of natural hybrids of gibel carp
Carassius auratus gibelio (Bloch) and crucian carp Carassius carassius (L.) from lower Dyje River floodplain (Czech Republic)," Journal of Fish Biology, vol. 72, no. 5, pp. 1230-1235, 2008.

[38] H. Liu, C.-S. Tzeng, and H.-Y. Teng, "Sequence variations in the mitochondrial DNA control region and their implications for the phylogeny of the Cypriniformes," Canadian Journal of Zoology, vol. 80, no. 3, pp. 569-581, 2002.

[39] K. Tamura, G. Stecher, D. Peterson, A. Filipski, and S. Kumar, "MEGA6: Molecular Evolutionary Genetics Analysis version 6.0," Molecular Biology and Evolution, vol. 30, no. 12, pp. 27252729, 2013.

[40] M. Clement, D. Posada, and K. A. Crandall, "TCS: A computer program to estimate gene genealogies," Molecular Ecology, vol. 9, no. 10, pp. 1657-1659, 2000.

[41] W. D. Hamilton, "Sex versus Non-Sex versus Parasite," Oikos, vol. 35, no. 2, p. 282, 1980.

[42] W. D. Hamilton, R. Axelrod, and R. Tanese, "Sexual reproduction as an adaptation to resist parasites (A review)," Proceedings of the National Acadamy of Sciences of the United States of America, vol. 87, no. 9, pp. 3566-3573, 1990.

[43] M. Tobler and I. Schlupp, "Parasites in sexual and asexual mollies (Poecilia, Poeciliidae, Teleostei): A case for the Red Queen?" Biology Letters, vol. 1, no. 2, pp. 166-168, 2005. 


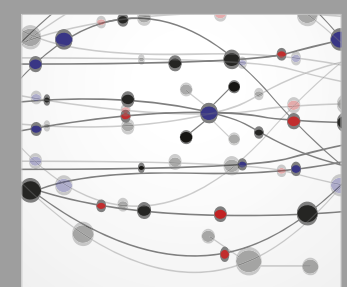

The Scientific World Journal
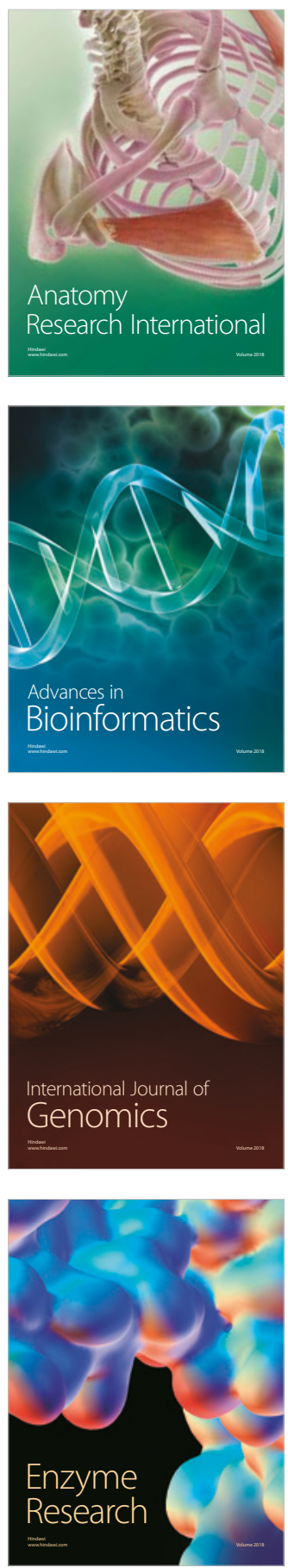
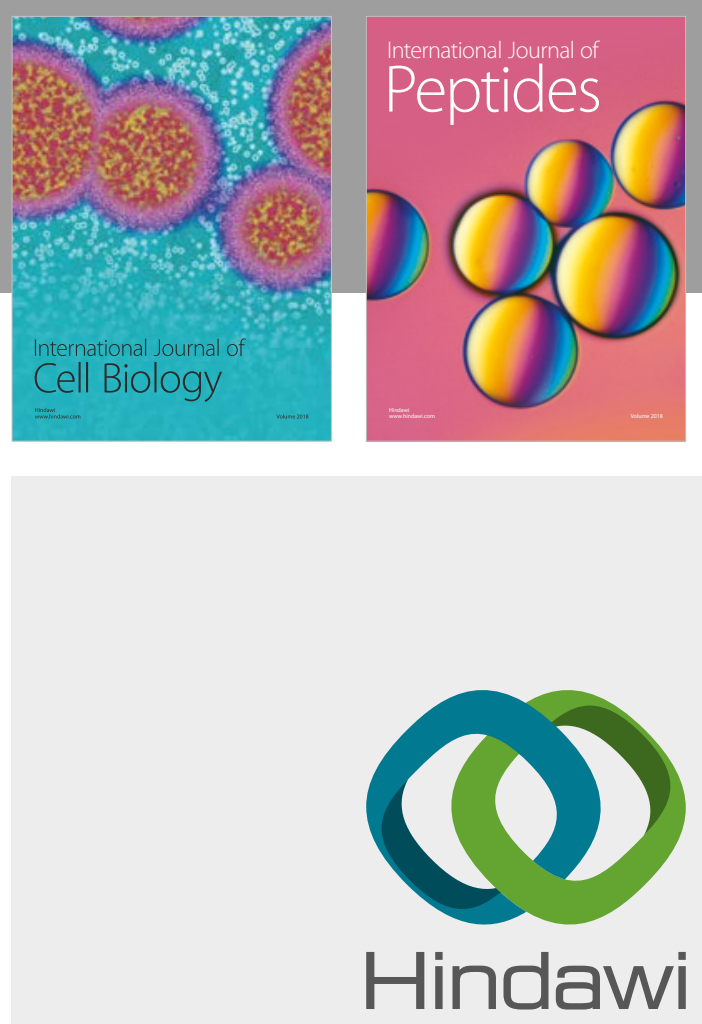

Submit your manuscripts at

www.hindawi.com
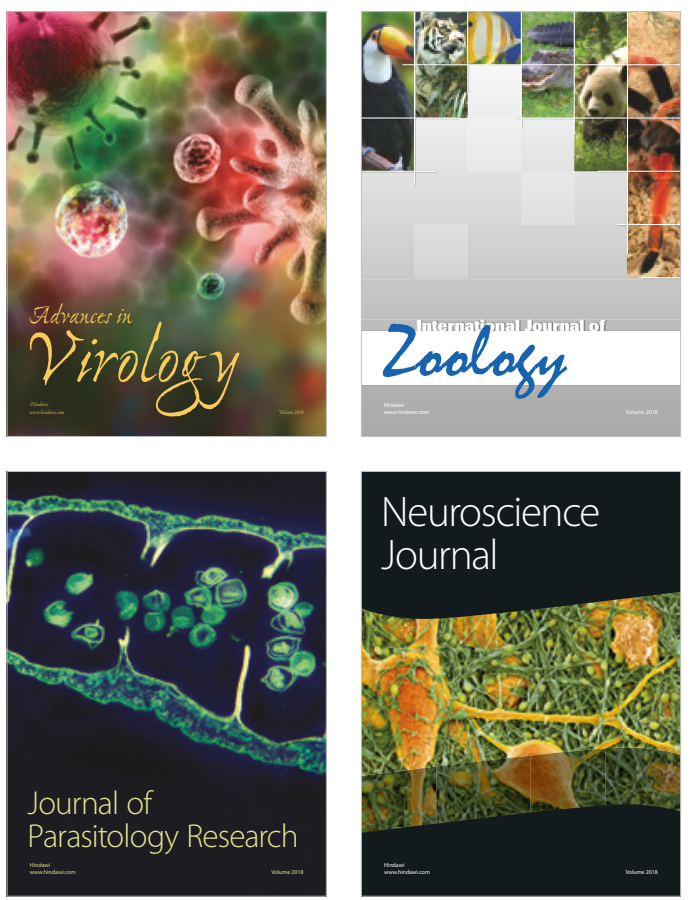
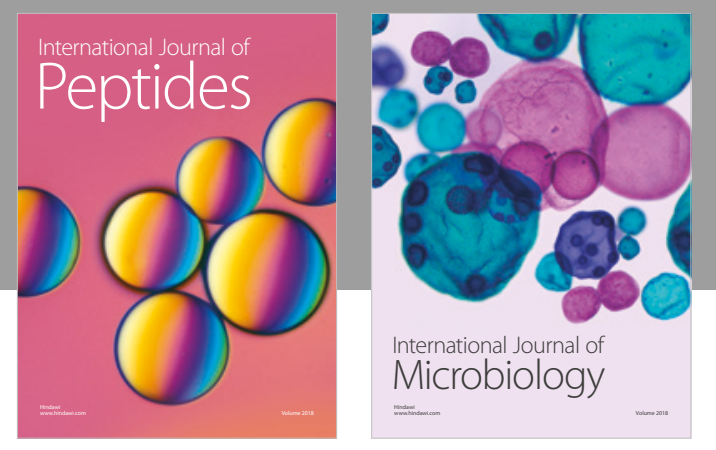

nternational Journal of Microbiology
Journal of
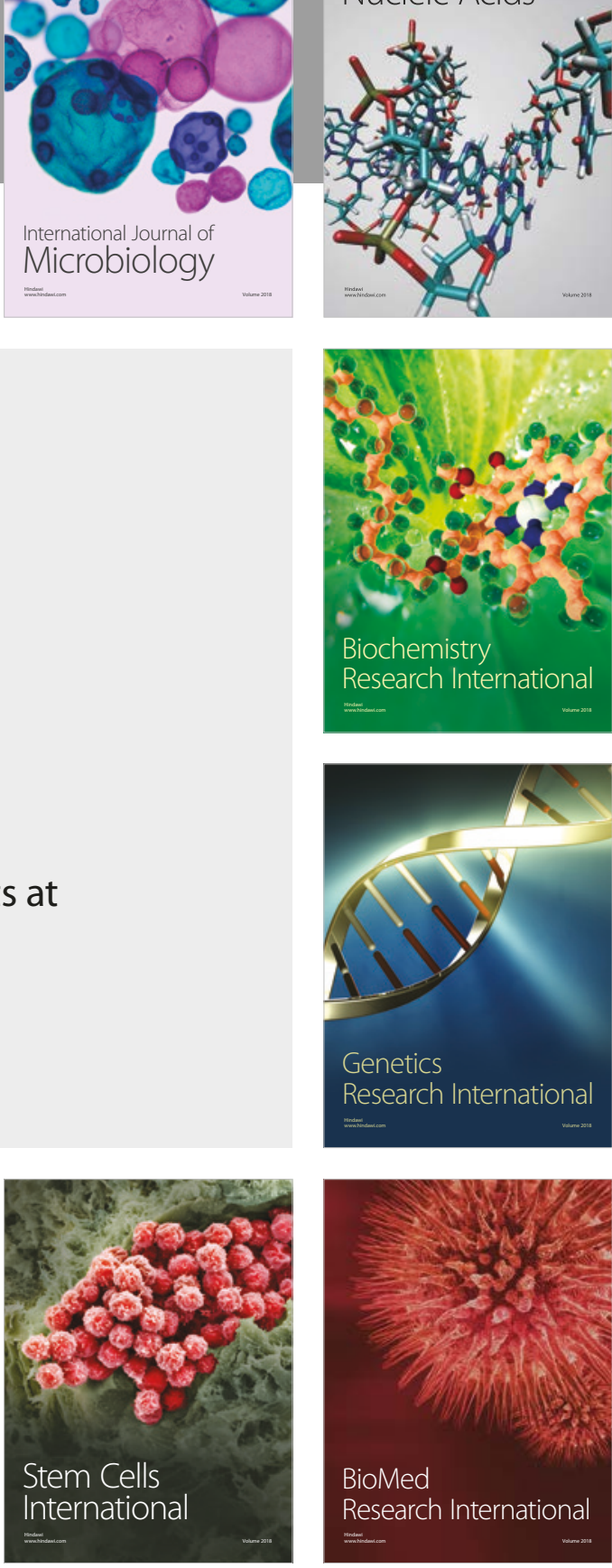
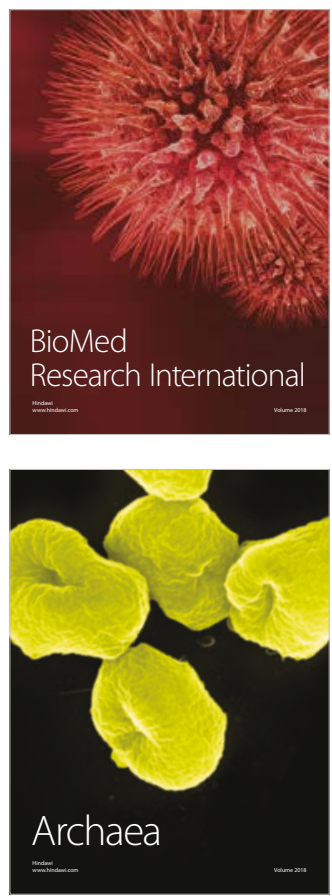